\title{
Meta-Analysis of Diffusion Metrics for the Prediction of Tumor Grade in Gliomas
}

\author{
V.Z. Miloushev, D.S. Chow, and C.G. Filippi
}

\begin{abstract}
BACKGROUND AND PURPOSE: Diffusion tensor metrics are potential in vivo quantitative neuroimaging biomarkers for the characterization of brain tumor subtype. This meta-analysis analyzes the ability of mean diffusivity and fractional anisotropy to distinguish lowgrade from high-grade gliomas in the identifiable tumor core and the region of peripheral edema.
\end{abstract}

MATERIALS AND METHODS: A meta-analysis of articles with mean diffusivity and fractional anisotropy data for World Health Organization low-grade (I, II) and high-grade (III, IV) gliomas, between 2000 and 2013, was performed. Pooled data were analyzed by using the odds ratio and mean difference. Receiver operating characteristic analysis was performed for patient-level data.

RESULTS: The minimum mean diffusivity of high-grade gliomas was decreased compared with low-grade gliomas. High-grade gliomas had decreased average mean diffusivity values compared with low-grade gliomas in the tumor core and increased average mean diffusivity values in the peripheral region. High-grade gliomas had increased FA values compared with low-grade gliomas in the tumor core, decreased values in the peripheral region, and a decreased fractional anisotropy difference between the tumor core and peripheral region.

CONCLUSIONS: The minimum mean diffusivity differs significantly with respect to the World Health Organization grade of gliomas. Statistically significant effects of tumor grade on average mean diffusivity and fractional anisotropy were observed, supporting the concept that high-grade tumors are more destructive and infiltrative than low-grade tumors. Considerable heterogeneity within the literature may be due to systematic factors in addition to underlying lesion heterogeneity.

ABBREVIATIONS: $\triangle F A=$ fractional anisotropy difference; $F A=$ fractional anisotropy; $M D=$ mean diffusivity; minMD = minimum mean diffusivity or minimum $A D C ; R O C=$ receiver operator characteristic; $W H O=$ World Health Organization

$D^{\text {s }}$ iffusion tensor imaging is an MR imaging technique that can quantify diffusion of water in the brain and characterize the structural integrity of white matter tracts. ${ }^{1-3}$ Multiple studies have examined the ability of basic diffusion tensor metrics such as mean diffusivity (MD) or the apparent diffusion coefficient and fractional anisotropy (FA) to discriminate the tumor grade of gliomas. Disruption of normal white matter structural integrity by primary glial neoplasms should theoretically reduce fractional anisotropy and increase mean diffusivity.

Mean diffusivity is positively correlated with decreased tumor cellular density and increased patient survival, and significant ef-

Received May 8, 2014; accepted after revision July 17.

From the Department of Diagnostic Radiology, Columbia University, New York, New York. Paper previously presented at: Mini-Symposiums on Tumor and Stroke at the Annual Meeting of the American Society of Neuroradiology and the Foundation of the ASNR Symposium, May 17-22, 2014; Montreal, Quebec, Canada.

Please address correspondence to Vesselin Z. Miloushev, MD, Department of Diagnostic Radiology, Columbia University, 622 West 168th St, PB 1-302C, New York, NY 10032; e-mail: vzm1@columbia.edu

http://dx.doi.org/10.3174/ajnr.A4097 fects are reported in several studies with respect to discriminating tumor grade specifically by using minimum mean diffusivity (minMD) ${ }^{4-9}$ In contradistinction, there is no definitive consensus on the ability of fractional anisotropy to assess tumor grade, cellular density, and parenchymal infiltration or to prognosticate patient survival. ${ }^{7,10-21}$ We performed a quantitative meta-analysis of the existing literature to determine the statistical consensus of mean diffusivity and fractional anisotropy in distinguishing tumor grade of gliomas, separately examining the identifiable tumor core and region of peripheral signal abnormality.

\section{MATERIALS AND METHODS}

Articles were identified via PubMed and Science Citation Index query using the terms "diffusion" and "brain tumor." This search produced 1657 articles from PubMed and 2158 articles from the Science Citation Index. Citations were imported into the EndNote citation manager (Thomson Reuters, New York, New York), which was used to remove duplicates, yielding 3128 citations. Articles were then restricted to those with publication dates between 2000 and 2013 and containing the word "glioma," which yielded 
Study characteristics and technical factors ${ }^{a}$

\begin{tabular}{lcc}
\hline \multicolumn{1}{c}{ Attribute } & MD & FA \\
\hline $\begin{array}{l}\text { Average no. of patients per tumor grade } \\
\quad \text { category }\end{array}$ & $15.3 \pm 12.0$ & $13.4 \pm 8.6$ \\
Average age of patients (yr) & $49.3 \pm 8.6$ & $50.0 \pm 7.6$ \\
Prospective design & $79.1 \%$ & $69.4 \%$ \\
Studies at 3T & $34.5 \%$ & $41.7 \%$ \\
Average maximum diffusion b-value & $1103 \pm 454$ & $111 \pm 448$ \\
Average no. of noncollinear directions & - & $21 \pm 31$ \\
\hline
\end{tabular}

Note:-indicates not calculated.

${ }^{a}$ SDs are reported for average values.

377 articles. An additional restriction to articles containing the phrase "fractional anisotropy" resulted in 242 articles. All studies (377 for mean diffusivity, 242 for fractional anisotropy) were read for relevance. Only studies that reported data for adult patients with histologic confirmation of treatment-naïve lesions were included. We could not control for sampling error associated with histologic sampling; with the exception of a few studies that performed stereotactic biopsies, it is possible that some lesions were inappropriately classified. ${ }^{22}$ Case reports were excluded.

FA and MD values were tabulated as mean values and SDs. The SDs and number of patients were used for weighting in the pooled analysis. Two articles displayed data in chart rather than numeric format; the chart images were analyzed by superimposing a finely decimated grid, which intersected the chart axis in the PowerPoint image manager (Microsoft, Redmond, Washington) to extract numeric values.

The World Health Organization (WHO) tumor grade and the range of histologic tumor types included were tabulated. Information on whether each study was prospective and/or retrospective, the number of patients, and the mean patient age, if provided, were recorded. The technical specifications for the diffusion acquisition, including main magnetic field strength, number of noncollinear gradient directions, number of b-values, and maximum b-value, were recorded. The MR imaging vendor and software used for analysis were noted.

We only included studies that separated diffusion metrics in the tumor core and tumor periphery, with the exception of 2 studies that reported the minimum mean diffusivity and included the entire region of signal abnormality. ${ }^{7,23}$ Studies that reported central necrotic regions for either tumor grade were excluded. Some studies separated tumor core values for enhancing and nonenhancing components, and these were recorded. Studies that reported values for the region of signal abnormality peripheral to the tumor core as either "edematous" or "infiltrated" were grouped into the peripheral region category; this was equated to the region of T2-prolongation on long-TR images, such as T2weighted or FLAIR images. Critically, the peripheral region was distinguished from the "intermediary" or "boundary" region between the tumor core and the peripheral region, reported in some studies in the neighborhood of 1-2 mm from the tumor core. Also relevant for low-grade lesions, data from studies that only reported the white matter adjacent to the region of signal abnormality were not included. ${ }^{24,25}$ Summary statistics for the studies are provided in the Table.

Equations relating MD, equivalent to the apparent diffusion coefficient, and FA are provided below in terms of the 3 principal eigenvalues $\left(\lambda_{1}, \lambda_{2}, \lambda_{3}\right) .{ }^{26}$ However, 3 noncollinear diffusion gra- dient directions suffice to calculate the mean diffusivity, without calculation of the individual eigenvalues. Adjustments were made if studies reported the trace instead of MD (trace $=3 \mathrm{MD})$.

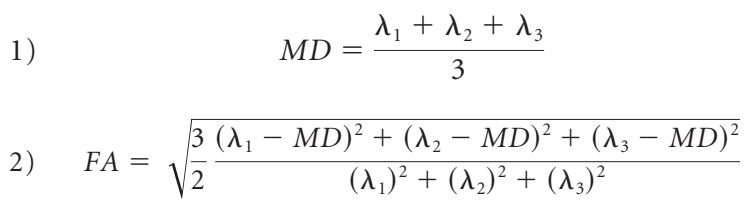

Statistical analysis was performed with R, Version 3.0.1 (http:// www.r-project.org). ${ }^{27}$ The metafor package (http://cran.rproject.org/web/packages/metafor/index.html) was used to implement a random-effects model, calculate $\mathrm{I}^{2}$ as a measure of heterogeneity, perform meta-regression, and generate forest plots. ${ }^{28}$ Standardized mean differences of mean diffusivity and fractional anisotropy between high-grade and low-grade gliomas were converted to odds ratios to simplify interpretation. ${ }^{29}$ The mean difference was used to calculate the difference in fractional anisotropy $(\Delta \mathrm{FA})$ between the tumor core and peripheral region. The funnel plot asymmetry regression test was used to evaluate study sample size bias. ${ }^{30}$ Approximate permutation tests for $P$ values used 1000 iterations. ${ }^{31}$ The $p R O C$ package (http://cran.r-project. org/web/packages/pROC/index.html) was used to generate receiver operating characteristic (ROC) curves and calculate area under the curve via bootstrapping (10,000 replicates) for patientlevel data. ${ }^{32}$ The binormal method was used for ROC curve smoothing. Confidence intervals were calculated at the $95 \%$ significance level.

\section{RESULTS}

\section{Minimum MD}

Pooled analysis of minimum mean diffusivity (minMD) with respect to tumor grade was performed in 17 unique studies (772 patients) (Fig $1 A$ ). There was a significant effect of tumor grade (WHO I and II, III and IV) on minMD, with the higher tumor grade resulting in decreased minMD values $(P<.001)$. Funnel plot asymmetry was not significant $(P=.96)$. Considerable heterogeneity was present $\left(\mathrm{I}^{2}=93 \%\right)$. Meta-regression models showed no significant effects for patient age, year of publication, MR imaging vendor, and main magnetic field strength $(P>.05)$. Dichotomizing into high-grade (WHO III and IV) and low-grade (WHO grade I and II) groups was significant $(P<.001)$; the mean minMD of low-grade gliomas was $1.19 \pm 0.06 \mathrm{~mm}^{2} / \mathrm{s}$, and the difference between the low-grade and high-grade groups was $0.37 \pm 0.07 \mathrm{~mm}^{2} / \mathrm{s}$.

Patient-level data were available in 5 studies (105 patients) (Fig 1C). ROC analysis resulted in an area under the curve of 0.84 (95\% CI, 0.76-0.91). The optimal threshold to distinguish lowgrade and high-grade gliomas was $\operatorname{minMD}=0.98 \mathrm{~mm}^{2} / \mathrm{s}$, identified via the Youden Index. This threshold resulted in a specificity of $78.3 \%$ (95\% CI, $66.7 \%-88.3 \%)$ and a sensitivity of $77.8 \%(95 \%$ CI, $64.4 \%-88.9 \%)$.

\section{Average MD}

Pooled analysis of average values of MD was also performed for determination of tumor grade in the tumor core (26 studies, 996 patients) and the peripheral region of signal abnormality (10 studies, 207 patients) (Fig $2 A,-B$ ). The analysis was restricted to 

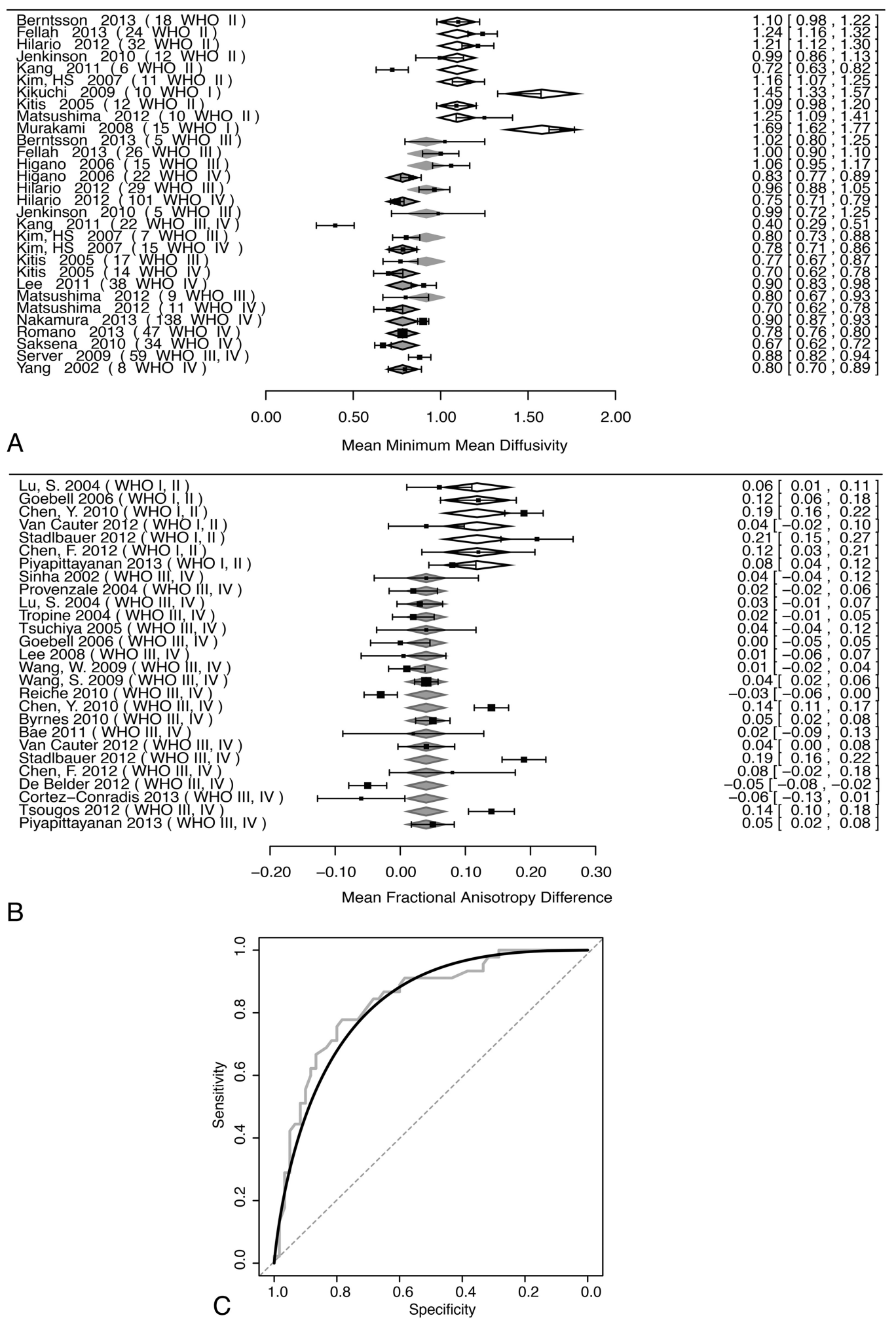

FIG 1. Effects of tumor grade on minimum mean diffusivity and $\Delta$ fractional anisotropy. Moderator analysis was performed ( $A$ and $B)$ with respect to tumor grade. In forest plots $(A$ and $B)$, the left column indexes each study by lead author and publication year, with the WHO tumor grade of the lesions in parentheses. WHO grade category means are shown by diamonds, with relative width corresponding to the standard error. The right column provides numeric mean values, with confidence intervals in brackets for each study. Pooled randomeffects values are provided at the bottom of each plot. A, The forest plot of minMD and effect of WHO tumor grade are shown. Open diamonds indicate WHO II; light-gray diamonds with gray borders, WHO III; dark gray diamonds with black borders, WHO IV. B, The forest plot of $\triangle$ FA is shown. Open diamonds indicate WHO I, II; light gray diamonds with gray borders, WHO III, IV. C, An ROC plot of patient-level data for minMD is shown. Gray step curve indicates actual data; black curve, binormal smoothed curve; dashed gray line, 50:50 line. 


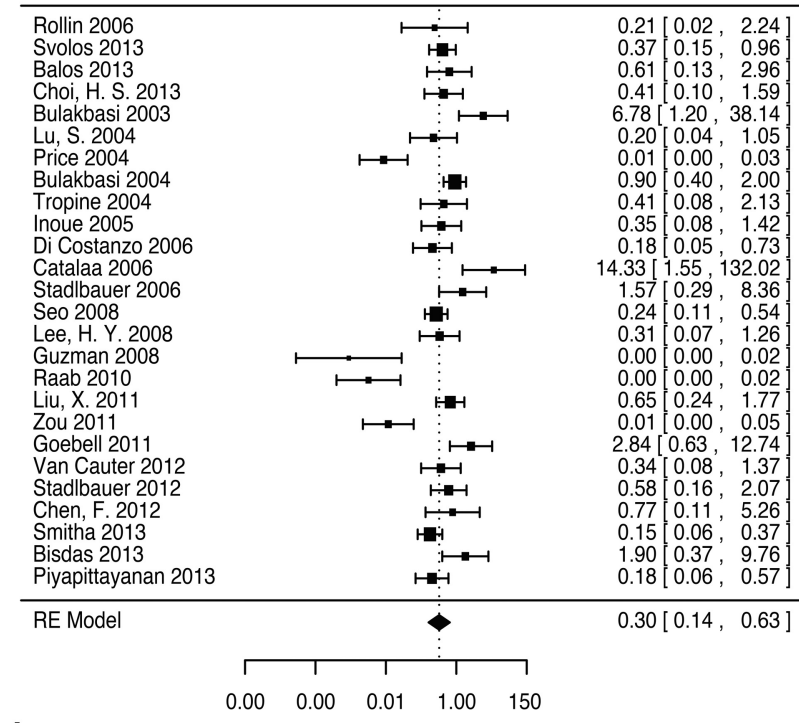

A Tumor Core Mean Diffusivity Odds Ratio (Log scale)

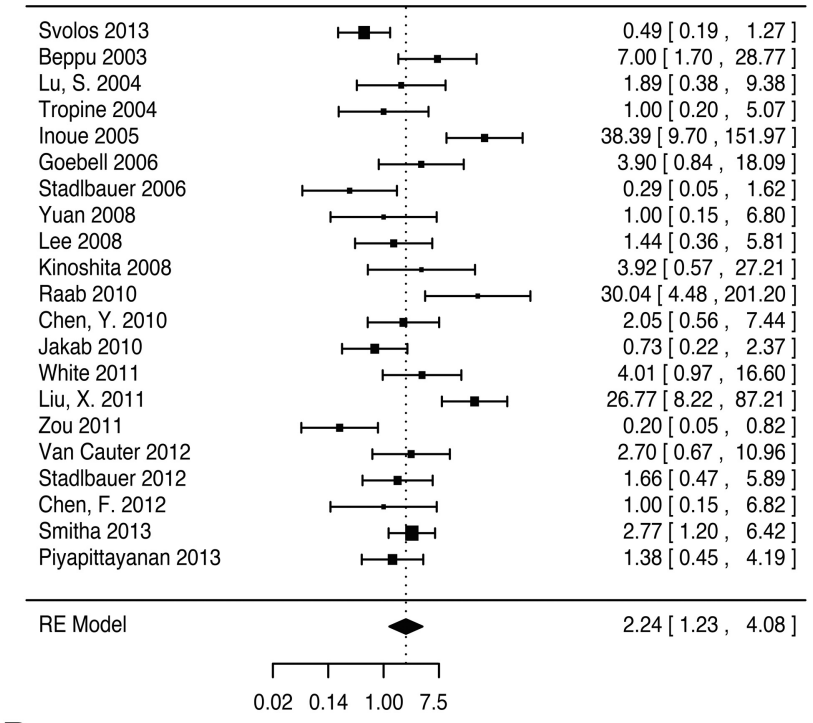

B

\begin{tabular}{|c|c|c|}
\hline $\begin{array}{l}\text { Lam } 2002 \\
\text { Lu, S. } 2004 \\
\text { Morita } 2005 \\
\text { Fan } 2006 \\
\text { Di Costanzo } 2006 \\
\text { Guzman } 2008 \\
\text { Van Cauter } 2012 \\
\text { Stadlbauer } 2012 \\
\text { Chen, F. 2012 } \\
\text { Piyapittayanan } 2013\end{array}$ & : & $\begin{array}{r}1.69[0.27,10.76] \\
1.77[0.36,8.77] \\
615.02[34.85,30852.78] \\
0.35[0.07,1.74] \\
24.64[1.90,320.48] \\
61.09[3.99,935.57] \\
1.17[0.05,25.35] \\
1.23[0.35,4.35] \\
2.92[0.41,20.72] \\
4.59[1.30,16.22]\end{array}$ \\
\hline RE Model & & $4.32[1.25,15.00]$ \\
\hline & $\begin{array}{c}1 \\
1.00\end{array}$ & \\
\hline
\end{tabular}

\begin{tabular}{|c|c|c|}
\hline $\begin{array}{l}\text { Lu, S. } 2004 \\
\text { Goebell } 2006 \\
\text { Chen, Y. } 2010 \\
\text { Van Cauter } 2012 \\
\text { Stadlbauer } 2012 \\
\text { Chen, F. } 2012 \\
\text { Piyapittayanan } 2013\end{array}$ & (1) & $\begin{array}{l}0.64[0.13,3.13] \\
0.13[0.03,0.64] \\
0.23[0.06,0.88] \\
2.48[0.11,56.15] \\
0.90[0.25,3.18] \\
0.52[0.07,3.58] \\
0.53[0.16,1.80]\end{array}$ \\
\hline RE Model & & $0.45[0.26,0.81]$ \\
\hline & $\begin{array}{ccc} & 1 & \\
0.02 & 0.14 & 1.00\end{array}$ & \\
\hline
\end{tabular}

FIG 2. Forest plots of mean diffusivity and fractional anisotropy in the tumor core and peripheral region of signal abnormality, comparing differences between low-grade and high-grade categories (moderator analysis was not performed). The standardized mean difference between high-grade and low-grade lesions was converted to odds ratios as a measure of effect size. Mean diffusivity in the tumor core $(A)$ and peripheral region $(B)$ with fractional anisotropy in the tumor core $(C)$ and peripheral region $(D)$ are shown. For each forest plot, the left column indexes each study by lead author and publication year. The right column provides odds ratios, with confidence intervals in brackets for each study. Pooled random-effect odds ratios are provided at the bottom of each plot.

studies that provided data for both low-grade and high-grade gliomas, to provide internal controls. The odds ratio for highgrade versus low-grade lesions was 0.3 (95\% CI, 0.14-0.63; permutation $P$ value $<.001)$ in the tumor core and $4.32(95 \% \mathrm{CI}$, 1.25-15.0; permutation $P$ value $=.044$ ) in the peripheral region; raw mean differences between high-grade and low-grade were, however, small ( -0.16 and 0.14 , respectively). Considerable significant heterogeneity was present for the tumor core, $\mathrm{I}^{2}=87.2 \%$ (95\% CI, 80.3\%-94.9\%), less significantly in the peripheral region, $\mathrm{I}^{2}=76.8 \%$ (95\% CI, 48.3\%-94.6\%).

\section{$\Delta \boldsymbol{F A}$}

Pooled analysis of the difference in fractional anisotropy $(\Delta \mathrm{FA})$ between the peripheral region of signal abnormality and the tumor core was performed in 20 unique studies (391 patients) (Fig $1 B)$. High-grade gliomas had a significantly decreased $\Delta \mathrm{FA}$ compared with low-grade gliomas $(P=.007)$. The raw difference estimate between the 2 groups was $0.08 \pm 0.03$ (estimated $\Delta \mathrm{FA}$ of low-grade gliomas $=0.12 \pm 0.03$ ). The permutation $P$ value remained significant $(P=.02)$, and the funnel plot asymmetry was not significant $(P=.6)$. Considerable heterogeneity was present, $\mathrm{I}^{2}=91 \%$ (95\% CI, 84.3\%-95.1\%). A meta-regression model incorporating MR imaging vendor type (GE Healthcare, Siemens, Philips Healthcare, Toshiba) was not significant $(P=.078)$; models incorporating patient age, year of publication, number of noncollinear DTI directions, and main magnetic field strength were also not significant.

\section{Average FA}

Pooled analysis of average values of FA was performed for determination of tumor grade in the tumor core (21 studies, 734 patients) and the peripheral region of signal abnormality ( 7 studies, 180 patients) (Fig $2 C,-D$ ). The analysis was restricted to studies that provided data for both low-grade and high-grade gliomas, to provide internal controls. The odds ratio for high-grade versus low-grade lesions was 2.24 (95\% CI, 1.23-4.08, permutation $P$ value $=.006)$ in the tumor core and 0.45 (95\% CI, 0.26-0.81, permutation $P$ value $=.032)$ in the peripheral region; raw mean differences between high-grade and low-grade were, however, small ( 0.02 and -0.02 , respectively). Modest heterogeneity was 
present for the tumor core, $\mathrm{I}^{2}=74.9 \%$ (95\% CI, 56.2\%-88.2\%), without significant heterogeneity in the peripheral region, $\mathrm{I}^{2}=$ 0\% (95\% CI, 0\%-82.8\%).

\section{DISCUSSION}

We performed a meta-analysis to explore the validity and consensus in the utility of mean diffusivity and fractional anisotropy for distinguishing tumor grade in gliomas. Pooled analysis was restricted to studies that internally compared low-grade and highgrade lesions, thus providing internal controls. Significant effects were observed, adding support to generalizations regarding tumor biology, though the raw effect sizes were small and significant heterogeneity was present in some of the cohorts of studies. In the identifiable tumor core, high-grade gliomas had decreased MD and increased FA values compared with low-grade gliomas. In the peripheral region of signal abnormality, high-grade gliomas had increased MD and decreased FA values. These observations suggest that high-grade gliomas have a more destructive effect on white matter tracts than low-grade gliomas in the peripheral region. In the tumor core, high-grade gliomas are expected to have increased extracellular-space volume and increased microvascular proliferation and are not expected to preserve white mater architecture, to account for the relatively greater fractional anisotropy. ${ }^{33,34}$ Theoretically, this effect may be a consequence of initial growth along the scaffold of white matter tracts. Alternatively, high-grade gliomas may have a less defined transition between the tumor core and periphery than is suggested by structural imaging.

We further analyzed the FA difference between the tumor core and peripheral region to provide additional insight into tumor biology. High-grade gliomas have a $\Delta \mathrm{FA}$ that is approximately $0.08 \pm 0.03$ smaller than that in low-grade gliomas. The result may suggest that high-grade gliomas are more infiltrative than low-grade gliomas, as expected from mathematic modeling. ${ }^{35}$ The results parallel those of Ferda et al, ${ }^{15}$ who observed that grade II gliomas have a sharper transition than grade III gliomas among the tumor core, intermediary region, and the peripheral region. We note, however, that their results also showed a sharp transition, presumably due to mass effect in grade IV gliomas, an observation that was not adequately testable in our meta-analysis.

The minMD was observed to be a significant diffusion imaging metric for distinguishing tumor grade in gliomas. While the minimum mean diffusivity is inherently subject to statistical noise and partial volume effects, it does not necessarily rely on precise lesion segmentation, thus eliminating a source of heterogeneity between studies. The ROC analysis of patient-level data suggested an optimal cutoff in minMD of $0.98 \mathrm{~mm}^{2} / \mathrm{s}$; minMD lower than this value favors a high-grade glioma. This suggested cutoff is within the range of previously published values. ${ }^{4-6}$ However, the lower bounds of the $95 \%$ CI for both sensitivity and specificity from our ROC analysis are approximately $65 \%$, which limits clinical confidence in using this metric alone.

Several limitations of our study are inherent in its methods. First, more significant differences may not have been observed simply because mean diffusivity and fractional anisotropy are insufficient to discriminate tumor grade. Unfortunately, promising metrics such as diffusional kurtosis, p:q diffusion tensor decom- position, and maximum SD of FA were provided in too few studies to be accessible by meta-analysis. ${ }^{19,36,37}$

Considerable heterogeneity was observed in the cohort of studies for some of the metrics we tested. One source of heterogeneity was sampling error in pathologic specimens used for histologic grading. This error is expected to increase heterogeneity in the dataset because high-grade lesions may be mistakenly classified as low-grade lesions; unfortunately because most studies did not perform multiple biopsies, it is not possible to control for this source of heterogeneity. Variations in measurement precision are unavoidable, though in individual patients, measurements of the fractional anisotropy and mean diffusivity showed good reproducibility in at least 1 study. ${ }^{38}$ However, measurement accuracy is difficult to account for among all studies. Nevertheless, technical factors such as the main magnetic field strength, MR imaging vendor, number of noncollinear diffusion gradient directions (in the case of FA), and number of b-values used were not found to significantly account for the heterogeneity among studies.

Nonquantitative aspects specifically related to segmentation of brain tumor components on imaging could not be adequately accounted for in our study. Discrimination of tumor components primarily relies on the expert opinion of neuroradiologists. Specific challenges arise for lesions lacking well-defined tumor core and peripheral region boundaries. Increased interest in semiautomated computer segmentation in the analysis of brain tumors, coupled with validation, may circumvent some subjectivity in delineating the image-definable components of glial tumors. ${ }^{39}$ Standardization of segmentation techniques is expected to improve the utility of quantitative measurements.

Furthermore, in most studies, a range of values was observed among patients. Sources of this variation include different tumor locations and underlying patient-specific background tissue differences. However, there is likely additional heterogeneity within the low-grade and high-grade glioma groups. Within glioblastoma, 4 separate subtypes can be distinguished by molecular profiling, and these subtypes differ in the degree of infiltration. ${ }^{40-42}$ For example, O6-methylguanine DNA methyltransferase promoter methylation has a significant effect on diffusion tensor metrics. ${ }^{43,44}$ WHO grade and single histologic designations are likely inadequate as sole descriptors of the biologic behavior of tumors.

\section{CONCLUSIONS}

Minimum mean diffusivity is an easily calculated diffusion tensor metric that differs significantly with respect to WHO tumor grade, though specific clinical recommendations cannot be made on the basis of this analysis. In the tumor core, high-grade gliomas have decreased MD and increased FA, while in the peripheral region high-grade gliomas have increased MD and decreased FA. However, considerable heterogeneity exists in the published literature, which is likely due to both systematic factors and the underlying biologic heterogeneity of gliomas. Standardization in terminology and segmentation of the regions of signal abnormality identifiable on imaging and standardization of DTI methodology are needed. However, to approach the underlying biologic heterogeneity of gliomas, future investigations may need to examine the correlation of diffusion tensor biomarkers with tumor genomic or expression profiles. Thus, diffusion tensor metrics can 
be tested as quantitative biomarkers for tumor subtype and can be potentially used to report subpopulations within a given tumor subtype.

Disclosures: Christopher G. Filippi-UNRELATED: Consultancy: Syntactx Corporation, Regeneration Pharmaceuticals, Comments: For both of these consultant jobs, I help them organize MR imaging protocols for clinical drug trials, and I interpret MR images; Grants/Grants Pending: Coinvestigator on National Institutes of Health/ National Cancer Institute (1R01CA161404-01A) and National Institutes of Health/National Heart, Lung, and Blood Institute (2R01HL071944-06).

\section{REFERENCES}

1. Hajnal JV, Doran M, Hall AS, et al. MR imaging of anisotropically restricted diffusion of water in the nervous system. J Comput Assist Tomogr 1991;15:1-18

2. Pierpaoli C, Jezzard P, Basser PJ, et al. Diffusion tensor MR imaging of the human brain. Radiology 1996;201:637-48

3. Basser PJ, Pierpaoli C. Microstructural and physiological features of tissues elucidated by quantitative-diffusion-tensor MRI. J Magn Reson B 1996;111:209-19

4. Higano S, Yun X, Kumabe T, et al. Malignant astrocytic tumors: clinical importance of apparent diffusion coefficient in prediction of grade and prognosis. Radiology 2006;241:839-46

5. Murakami R, Hirai T, Kitajima M, et al. Magnetic resonance imaging of pilocytic astrocytomas: usefulness of the minimum apparent diffusion coefficient (ADC) value for differentiation from high-grade gliomas. Acta Radiol 2008;49:462-67

6. Yamasaki F, Kurisu K, Aoki T, et al. Advantages of high b-value diffusion-weighted imaging to diagnose pseudo-responses in patients with recurrent glioma after bevacizumab treatment. Eur J Radiol 2012;81:2805-10

7. Saksena S, Jain R, Narang J, et al. Predicting survival in glioblastomas using diffusion tensor imaging metrics. J Magn Reson Imaging 2010;32:788-95

8. Zulfiqar M, Yousem DM, Lai H. ADC values and prognosis of malignant astrocytomas: does lower ADC predict a worse prognosis independent of grade of tumor? A meta-analysis. AJR Am J Roentgenol 2013;200:624-29

9. Chen L, Liu M, Bao J, et al. The correlation between apparent diffusion coefficient and tumor cellularity in patients: a meta-analysis. PLoS ONE 2013;8:e79008

10. Lu S, Ahn D, Johnson G, et al. Diffusion-tensor MR imaging of intracranial neoplasia and associated peritumoral edema: introduction of the tumor infiltration index. Radiology 2004;232:221-28

11. Beppu T, Inoue T, Shibata $Y$, et al. Fractional anisotropy value by diffusion tensor magnetic resonance imaging as a predictor of cell density and proliferation activity of glioblastomas. Surg Neurol 2005;63:56-61, discussion 61

12. Morita K, Matsuzawa H, Fujii Y, et al. Diffusion tensor analysis of peritumoral edema using lambda chart analysis indicative of the heterogeneity of the microstructure within edema. J Neurosurg 2005;102:336-41

13. Stadlbauer A, Ganslandt O, Buslei R, et al. Gliomas: histopathologic evaluation of changes in directionality and magnitude of water diffusion at diffusion-tensor MR imaging. Radiology 2006;240:803-10

14. Lee HY, Na DG, Song IC, et al. Diffusion-tensor imaging for glioma grading at 3-T magnetic resonance imaging: analysis of fractional anisotropy and mean diffusivity. J Comput Assist Tomogr 2008;32:298-303

15. Ferda J, Kastner J, Mukensnabl P, et al. Diffusion tensor magnetic resonance imaging of glial brain tumors. Eur J Radiol 2010;74:428-36

16. Kinoshita M, Goto T, Okita Y, et al. Diffusion tensor-based tumor infiltration index cannot discriminate vasogenic edema from tumor-infiltrated edema. J Neurooncol 2010;96:409-15

17. Deng Z, Yan Y, Zhong D, et al. Quantitative analysis of glioma cell invasion by diffusion tensor imaging. J Clin Neurosci 2010;17: 1530-36

18. Jakab A, Molnar P, Emri M, et al. Glioma grade assessment by using histogram analysis of diffusion tensor imaging-derived maps. $\mathrm{Neu}$ roradiology 2011;53:483-91

19. White ML, Zhang Y, Yu F, et al. Diffusion tensor MR imaging of cerebral gliomas: evaluating fractional anisotropy characteristics. AJNR Am J Neuroradiol 2011;32:374-81

20. Zikou AK, Alexiou GA, Kosta P, et al. Diffusion tensor and dynamic susceptibility contrast MRI in glioblastoma. Clin Neurol Neurosurg 2012;114:607-12

21. Sternberg EJ, Lipton ML, Burns J. Utility of diffusion tensor imaging in evaluation of the peritumoral region in patients with primary and metastatic brain tumors. AJNR Am J Neuroradiol 2014;35:439-44

22. Stadlbauer A, Nimsky C, Buslei R, et al. Diffusion tensor imaging and optimized fiber tracking in glioma patients: histopathologic evaluation of tumor-invaded white matter structures. Neuroimage 2007;34:949-56

23. Kang Y, Choi SH, Kim YJ, et al. Gliomas: histogram analysis of apparent diffusion coefficient maps with standard- or high-b-value diffusion-weighted MR imaging-correlation with tumor grade. Radiology 2011;261:882-90

24. Chen Y, Shi Y, Song Z. Differences in the architecture of low-grade and high-grade gliomas evaluated using fiber density index and fractional anisotropy. J Clin Neurosci 2010;17:824-29

25. Nilsson D, Rutka JT, Snead OC 3rd, et al. Preserved structural integrity of white matter adjacent to low-grade tumors. Childs Nerv Syst 2008:24:313-20

26. Kingsley $\mathrm{PB}$. Introduction to diffusion tensor imaging mathematics. Part II. Anisotropy, diffusion-weighting factors, and gradient encoding schemes. Concepts in Magnetic Resonance Part A 2006;28A:123-54

27. R Development Core Team. R: A Language and Environment for Statistical Computing. Version 3.0.1. Vienna, Austria: R Foundation for Statistical Computing; 2013

28. Viechtbauer W. Conducting meta-analyses in $\mathrm{R}$ with the metafor package. Journal of Statistical Software 2010;36:1-48

29. Chinn S. A simple method for converting an odds ratio to effect size for use in meta-analysis. Stat Med 2000;19:3127-31

30. Egger M, Davey Smith G, Schneider M, et al. Bias in meta-analysis detected by a simple, graphical test. BMJ 1997;315:629-34

31. Higgins JP, Thompson SG. Controlling the risk of spurious findings from meta-regression. Stat Med 2004;23:1663-82

32. Robin X, Turck N, Hainard A, et al. pROC: an open-source package for $\mathrm{R}$ and $\mathrm{S}+$ to analyze and compare ROC curves. BMC Bioinformatics 2011;12:77

33. Wesseling P, Vandersteenhoven J, Downey B, et al. Cellular components of microvascular proliferation in human glial and metastatic brain neoplasms. Acta Neuropathol 1993;85:508-14

34. Vargová L, Homola A, Zamecnik J, et al. Diffusion parameters of the extracellular space in human gliomas. Glia 2003;42:77-88

35. Swanson KR, Alvord EC Jr, Murray JD. A quantitative model for differential motility of gliomas in grey and white matter. Cell Prolif 2000;33:317-29

36. Raab P, Hattingen E, Franz K, et al. Cerebral gliomas: diffusional kurtosis imaging analysis of microstructural differences. Radiology 2010;254:876-81

37. Van Cauter S, Veraart J, Sijbers J, et al. Gliomas: diffusion kurtosis MR imaging in grading. Radiology 2012;263:492-501

38. Paldino MJ, Barboriak D, Desjardins A, et al. Repeatability of quantitative parameters derived from diffusion tensor imaging in patients with glioblastoma multiforme. J Magn Reson Imaging 2009;29:1199-205

39. Chow DS, Qi J, Guo X, et al. Semiautomated volumetric measurement on postcontrast MR imaging for analysis of recurrent and residual disease in glioblastoma multiforme. AJNR Am J Neuroradiol 2014;35:498-503

40. Phillips HS, Kharbanda S, Chen R, et al. Molecular subclasses of high-grade glioma predict prognosis, delineate a pattern of disease progression, and resemble stages in neurogenesis. Cancer Cell 2006;9:157-73

41. Zinn PO, Majadan B, Sathyan P, et al. Radiogenomic mapping of 
edema/cellular invasion MRI-phenotypes in glioblastoma multiforme. PLoS ONE 2011;6:e25451

42. Verhaak RG, Hoadley KA, Purdom E, et al. An integrated genomic analysis identifies clinically relevant subtypes of glioblastoma characterized by abnormalities in PDGFRA, IDH1, EGFR and NF1. Cancer Cell 2010;17:98

43. Moon WJ, Choi JW, Roh HG, et al. Imaging parameters of high grade gliomas in relation to the MGMT promoter methylation status: the CT, diffusion tensor imaging, and perfusion MR imaging. Neuroradiol 2012;54:555-63

44. Romano A, Calabria LF, Tavanti F, et al. Apparent diffusion coefficient obtained by magnetic resonance imaging as a prognostic marker in glioblastomas: correlation with MGMT promoter methylation status. Eur Radiol 2013;23:513-20 